\title{
ON THE HELMHOLTZ DECOMPOSITION FOR POLYADICS
}

\author{
BY \\ GEORGE DASSIOS (Division of Applied Mathematics, Department of Chemical Engineering, \\ University of Patras and ICEHT-FORTH, Greece) \\ AND \\ ISMO V. LINDELL (Electromagnetics Laboratory, Helsinki University of Technology, P.O. Box \\ 3000, FIN-02015HUT, Espoo, Finland)
}

\begin{abstract}
A polyadic field of rank $n$ is the tensor product of $n$ vector fields. Helmholtz showed that a vector field, which is a polyadic field of rank 1 , is nonuniquely decomposable into the gradient of a scalar function plus the rotation of a vector function. We show here that a polyadic field of rank $n$ is, again nonuniquely, decomposable into a term consisting of $n$ successive applications of the gradient to a scalar function, plus a term that consists of $(n-1)$ successive applications of the gradient to the rotation of a vector function, plus a term that consists of $(n-2)$ successive applications of the gradient to the rotation of the rotation of a dyadic function and so on, until the last $(n+1)$ th term, which consists of $n$ successive applications of the rotation operator to a polyadic function of rank $n$. Obviously, the $n=1$ case recovers the Helmholtz decomposition theorem. For dyadic fields a more symmetric representation is provided and formulae that provide the potential representation functions are given. The special cases of symmetric and antisymmetric dyadics are discussed in detail. Finally, the multidivergence type relations, which reduce the number of independent scalar representation functions to $n^{2}$, are presented.
\end{abstract}

1. Introduction. The representation of a vector field as the sum of the gradient of a scalar potential plus the rotation of a vector potential [1] has at least one use in almost every theory of physics, and the corresponding physical interpretation in each case is fundamental and basic. Considering the number of known physical quantities that have a dyadic structure, it is amazing why such a decomposition for dyadic fields has not been given before. This task was undertaken by the present authors and the extension of this decomposition property to a polyadic [1] of any rank is reported here.

Decomposition theorems of the Helmholtz type provide the starting point for most differential representations of vector fields, mainly in continuum mechanics and in other

Received February 21, 2000.

2000 Mathematics Subject Classification. Primary 35J05, 35J15, 35J25, 46L06.

Key words and phrases. Vector fields, dyadics, polyadics, Helmholtz deccmposition. 
physical realms as well. This is so since the gradient operator provides a natural way to incorporate all directional derivatives of scalar functions to one entity $[1,8]$, while the rotation operator is the vector invariant of the gradient of a vector field $[1,8]$. Both of these fundamental properties have direct physical analogies in continuum mechanics. Differential representations of the Papkovich-Neuber or the Boussinesq-Galerkin type [2, $4,6,7,9,16,18,19,20,21]$ for the displacement field in elasticity are based on the action of the gradient and the rotation operator on harmonic or biharmonic functions. The same is true for the representation of the velocity and the pressure fields in Stokes flow where similar representations have been proposed $[10,12,17,22,23]$. For electromagnetism, extended use of polyadic fields and their representations can be found in [13], while in [11, 14] differential representations for angular momentum with direct physical interpretation are provided. Physically dictated decompositions for Cartesian dyadics that appear in gravitational fields are given in [15]. Interesting results associated with the question of uniqueness can be found in [24]. The functional analytic approach to decomposition theorems for vector fields is given in [3].

The work at hand has its origin in our effort to unify the theory of acoustic, electromagnetic and elastic scattering into a single theory of dyadic scattering [5]. Such a theory recovers acoustic, electromagnetic and elastic scattering as special cases and at the same time reveals all possibilities that are hidden for scalar and vector fields [5].

Section 2 contains a detailed analysis of the dyadic case, which has more interest, from the point of view of applications, than the general polyadic fields. The decomposition formula for polyadic fields is given in Sec. 3 .

\section{Decomposition of dyadics.}

2.1. Helmholtz decomposition. Let $\Omega$ be a connected, open set of $\mathbb{R}^{3}$ and let $\bar{\Omega}=$ $\Omega \cup \partial \Omega$, where $\partial \Omega$ denotes the boundary of $\Omega$. A vector field $\mathbf{f}$, which is smooth in the sense that it is continuous on $\bar{\Omega}$ and possesses continuous first derivatives in $\Omega$, is called irrotational when

$$
\nabla \times \mathbf{f}(\mathbf{r})=\mathbf{0}, \quad \mathbf{r} \in \Omega .
$$

Such a ficld admits a representation of the form

$$
\mathbf{f}(\mathbf{r})=\nabla \phi(\mathbf{r}), \quad \mathbf{r} \in \Omega,
$$

for some $C^{1}$ scalar function $\phi$ known as the scalar potential [1,9]. In other words, if the vector ficld does not cause any rotation, its vectorial character is represented by the gradient operator.

On the other hand, $\mathbf{f}$ is called solenoidal when it satisfies

$$
\nabla \cdot \mathbf{f}(\mathbf{r})=0, \quad \mathbf{r} \in \Omega
$$

and such a solenoidal field admits a representation of the form

$$
\mathbf{f}(\mathbf{r})=\nabla \times \mathbf{A}(\mathbf{r}), \quad \mathbf{r} \in \Omega,
$$

for some $C^{1}$ vector function $\mathbf{A}$ known as the vector potential [1,9]. This terminology has its origin in fluid mechanics, where the velocity field for a fluid flowing through a 
pipe (in greek solena) enjoys a vanishing divergence due to the lack of sources or sinks. Therefore, if the vector field exists in the absence of sources or sinks, then its vectorial character represents rotation.

Helmholtz, in his celebrated decomposition theorem [9], showed that the above two properties of vector fields are the only possible ones. In fact, he proved that, if $\mathbf{f}$ is any vector field, such that $\mathbf{f} \in C^{1}(\Omega) \cap C(\bar{\Omega})$, it can be decomposed as

$$
\mathbf{f}(\mathbf{r})=\mathbf{f}^{i}(\mathbf{r})+\mathbf{f}^{s}(\mathbf{r})
$$

so that $\mathbf{f}^{i}$ is irrotational and $\mathbf{f}^{s}$ is solenoidal.

Consequently, given any smooth vector field $\mathbf{f}$, there exists a scalar potential $\phi$ and a vector potential $\mathbf{A}$ so that

$$
\mathbf{f}(\mathbf{r})=\nabla \phi(\mathbf{r})+\nabla \times \mathbf{A}(\mathbf{r})
$$

Obviously, $\phi$ and $\mathbf{A}$ are not uniquely defined in terms of $\mathbf{f}^{i}$ and $\mathbf{f}^{s}$.

In particular, $\phi$ can be obtained from a line integral of $\mathbf{f}^{i}[1]$ and it is uniquely defined up to an additive constant, while $\mathbf{A}$ can be obtained from a line integral of $\mathbf{f}^{s}$ [1] and it is uniquely defined up to an additive gradient of a harmonic function. Thus, $\phi$ can be replaced by

$$
\phi^{\prime}(\mathbf{r})=\phi(\mathbf{r})+c
$$

where $c$ is any constant and $\mathbf{A}$ can be replaced by

$$
\mathbf{A}^{\prime}(\mathbf{r})=\mathbf{A}(\mathbf{r})+\nabla u(\mathbf{r})
$$

where $u$ is any harmonic function, keeping the representation (6) invariant.

Furthermore, the irrotational part of the vector potential A does not contribute anything to the representation (6) [13] and therefore we can assume that

$$
\nabla \cdot \mathbf{A}(\mathbf{r})=0 .
$$

In other words, the vector potential can be taken to be solenoidal. Condition (9) can be utilized to express the solenoidal part of the field as successive applications of the rotation operator on a vector potential as long as the vector potential is smooth enough. That is,

$$
\mathbf{f}^{s}(\mathbf{r})=\nabla \times \nabla \times \cdots \times \nabla \times \mathbf{A}^{\prime}(\mathbf{r})
$$

for some smooth potential $\mathbf{A}^{\prime}(\mathbf{r})$.

The Helmholtz decomposition (6) became the king in the realm of Mathematical Physics, since this mathematical decomposition reflects basic physical characteristics of the vector fields involved in many disciplines of physics such as Electromagnetics, Fluid Mechanics, Elasticity, and so on. For example, in the classical theory of elasticity $[2,9,21]$, the irrotational part of the displacement field corresponds to the longitudinal (dilational) deformations and the solenoidal part of the displacement field corresponds to the transverse (shear) deformations, and these two kinds of deformations are the only ones possible within this theory. A scalar potential is needed to describe the dilation or the compression of the medium and a vector solenoidal potential is needed to describe its shear distortion. Note that (9) reduces the number of independent scalar functions 
to three, one for $\phi$ and two for $\mathbf{A}$, to exactly as many independent potentials as there are components in the field $\mathbf{f}$.

2.2. Decomposition of dyadics. Utilizing linearity, the above Helmholtz decomposition can be extended to smooth dyadic fields as follows. It is known [1] that any dyadic field $\widetilde{\mathbf{F}}$ in $\mathbb{R}^{3}$ enjoys the representation

$$
\widetilde{\mathbf{F}}(\mathbf{r})=\sum_{n=1}^{3} \mathbf{g}_{n}(\mathbf{r}) \otimes \mathbf{h}_{n}(\mathbf{r})
$$

for some vector fields $\mathbf{f}_{n}, \mathbf{g}_{n}, n=1,2,3$. In the Cartesian system $\hat{\mathbf{x}}_{i}, i=1,2,3,(11)$ is written as

$$
\begin{aligned}
\widetilde{\mathbf{F}}(\mathbf{r}) & =\sum_{n=1}^{3} \mathbf{g}_{n}(\mathbf{r}) \otimes\left(\sum_{i=1}^{3} \mathbf{h}_{n}(\mathbf{r}) \cdot \hat{\mathbf{x}}_{i} \otimes \hat{\mathbf{x}}_{i}\right) \\
& =\sum_{i=1}^{3}\left(\sum_{n=1}^{3} \mathbf{g}_{n}(\mathbf{r}) \otimes \mathbf{h}_{n}(\mathbf{r})\right) \cdot \hat{\mathbf{x}}_{i} \otimes \hat{\mathbf{x}}_{i} \\
& =\sum_{i=1}^{3} \mathbf{f}_{i}(\mathbf{r}) \otimes \hat{\mathbf{x}}_{i},
\end{aligned}
$$

where the vector fields $\mathbf{f}_{i}(\mathbf{r})$ are given by

$$
\mathbf{f}_{i}(\mathbf{r})=\sum_{n=1}^{3}\left[\mathbf{g}_{n}(\mathbf{r}) \otimes \mathbf{h}_{n}(\mathbf{r})\right] \cdot \hat{\mathbf{x}}_{i}, \quad i=1,2,3 .
$$

The Helmholtz decomposition secures that for each $i=1,2,3$ there exists a scalar potential $\phi_{i}$ and a vector potential $\mathbf{A}_{i}$ such that

$$
\mathbf{f}_{i}(\mathbf{r})=\nabla \phi_{i}(\mathbf{r})+\nabla \times \mathbf{A}_{i}(\mathbf{r})
$$

Substituting (14) back into (12), we arrive at the representation

$$
\begin{aligned}
\widetilde{\mathbf{F}}(\mathbf{r}) & =\sum_{i=1}^{3}\left[\nabla \phi_{i}(\mathbf{r})+\nabla \times \mathbf{A}_{i}(\mathbf{r})\right] \otimes \hat{\mathbf{x}}_{i} \\
& =\sum_{i=1}^{3} \nabla \otimes\left[\phi_{i}(\mathbf{r}) \hat{\mathbf{x}}_{i}\right]+\sum_{i=1}^{3} \nabla \times\left[\mathbf{A}_{i}(\mathbf{r}) \otimes \hat{\mathbf{x}}_{i}\right] .
\end{aligned}
$$

Next we define the vector potential

$$
\boldsymbol{\Phi}(\mathbf{r})=\sum_{i=1}^{3} \phi_{i}(\mathbf{r}) \hat{\mathbf{x}}_{i}
$$

and the dyadic potential

$$
\widetilde{\mathbf{A}}(\mathbf{r})=\sum_{i=1}^{3} \mathbf{A}_{i}(\mathbf{r}) \otimes \hat{\mathbf{x}}_{i}
$$

in terms of which the dyadic field $\widetilde{\mathbf{F}}$ assumes the decomposition

$$
\tilde{\mathbf{F}}(\mathbf{r})=\nabla \otimes \boldsymbol{\Phi}(\mathbf{r})+\nabla \times \tilde{\mathbf{A}}(\mathbf{r}) .
$$


Obviously, the three conditions

$$
\nabla \cdot \mathbf{A}_{i}(\mathbf{r})=0, \quad i=1,2,3,
$$

do not restrict the generality of the representation (18). The nine components of $\widetilde{\mathbf{F}}$ are now represented by the three components of $\phi$ and the six independent components of $\widetilde{\mathbf{A}}$. The vector potential $\mathbf{\Phi}$ accepts a decomposition of the form (6) and the dyadic potential $\widetilde{\mathbf{A}}$ accepts a representation of the form (18). Combining these representations with the fact that the gradient of the scalar field is irrotational, we arrive at the representation

$$
\widetilde{\mathbf{F}}(\mathbf{r})=\nabla \otimes \nabla \phi(\mathbf{r})+\nabla \otimes \nabla \times \mathbf{B}(\mathbf{r})+\nabla \times \nabla \times \widetilde{\mathbf{A}}(\mathbf{r})
$$

for some scalar potential $\phi$, some vector potential $\mathbf{B}$, and some dyadic potential $\widetilde{\mathbf{A}}$.

The representation (20) combines all possibilities of the gradient and rotation operators. It involves the gradient of the gradient of a scalar, the gradient of the rotation of a vector, and the rotation of the rotation of a dyadic. Observe that the dyadic character of the field $\widetilde{\mathbf{F}}$ in each term of the representation (20) is expressed either through the vectorial character of the gradient or through the polyadic rank of the potential. As we move down the line of the right-hand side of (20), each gradient is replaced by an ordered vector of the corresponding potential, and the representation starts with ordered gradients and ends with a potential that has the same rank as the initial field $\widetilde{\mathbf{F}}$.

If we interpret these in terms of classical elasticity, we see that any dyadic displacement field can be decomposed as two successive dilations (grad-grad term), two successive rotations (curl-curl term), and a rotation succeeded by a dilation (grad-curl term).

Note that $\widetilde{\mathbf{A}}$ is itself a dyadic field and therefore it has a representation of the form $(20)$ :

$$
\widetilde{\mathbf{A}}(\mathbf{r})=\nabla \otimes \nabla \phi^{\prime}(\mathbf{r})+\nabla \otimes \nabla \times \mathbf{B}^{\prime}(\mathbf{r})+\nabla \times \nabla \times \widetilde{\mathbf{A}}^{\prime}(\mathbf{r}) .
$$

But if we take the curl-operation of (21), we see that only the last term survives. Hence, without loss of generality, we can consider a dyadic field $\widetilde{\mathbf{A}}$ that accepts the representation (21) with

$$
\nabla \phi^{\prime}(\mathbf{r})=\mathbf{0}
$$

and

$$
\nabla \times \mathbf{B}^{\prime}(\mathbf{r})=\mathbf{0}
$$

In other words, the dyadic potential $\widetilde{\mathbf{A}}$ does not need to have any term that involves a gradient.

The field $\widetilde{\mathbf{F}}$ has nine scalar components. The representation involves $1+3+9=13$ scalar potentials, which are connected via the $1+3=4$ conditions (22) and (23). Hence, only 9 of the 13 potentials are independent.

In order to express restrictions (22) and (23) as conditions on $\widetilde{\mathbf{A}}$, we remark that what we actually need from (21) is that only the last term on the right-hand side survives and this is secured if we demand that

$$
\nabla \cdot \widetilde{\mathbf{A}}(\mathbf{r})=\mathbf{0}
$$


and

$$
\nabla \otimes \nabla: \tilde{\mathbf{A}}(\mathbf{r})=0
$$

where the double contraction is defined as [8]

$$
(\mathbf{a} \otimes \mathbf{b}):(\mathbf{c} \otimes \mathbf{d})=(\mathbf{a} \cdot \mathbf{c})(\mathbf{b} \cdot \mathbf{d}) .
$$

2.3. Symmetric decomposition of dyadics. The representation (20) is complete but it lacks symmetry. Making use of the transpose operator would give us another form where the gradient and rotation operators are operating from the right, like a mirror image of (20). It is possible to express the decomposition in a symmetric form not different from its mirror image. Let us assume that the gradient and rotation operators can operate from both sides on the potential functions. Operation from the left is emphasized by the symbol $\overleftarrow{\nabla}$.

To find the decomposition of a given dyadic field $\widetilde{\mathbf{F}}$ let us assume that there is a dyadic field $\widetilde{\mathbf{H}}$ that solves the fourth-order equation

$$
\nabla^{4} \widetilde{\mathbf{H}}(\mathbf{r})=\Delta^{2} \widetilde{\mathbf{H}}(\mathbf{r})=\widetilde{\mathbf{F}}(\mathbf{r}) .
$$

The fields $\widetilde{\mathbf{H}}$ can be made unique with suitable additional (boundary) conditions [24]. With the agreement above we can write Eq. (27) in the form

$$
\nabla^{2} \widetilde{\mathbf{I}} \cdot \widetilde{\mathbf{H}}(\mathbf{r}) \cdot \widetilde{\mathbf{I}} \bar{\nabla}^{2}=\widetilde{\mathbf{F}}(\mathbf{r})
$$

where $\widetilde{\mathbf{I}}$ stands for the unit dyadic (idemfactor). Now we can write the dyadic Laplace operator in two different ways as follows:

$$
\nabla^{2} \widetilde{\mathbf{I}}=\nabla \otimes \nabla-\nabla \times(\nabla \times \widetilde{\mathbf{I}})=\nabla \otimes \nabla-(\widetilde{\mathbf{I}} \times \nabla) \times \nabla
$$

Inserting these in (28) we obtain

$$
\widetilde{\mathbf{F}}(\mathbf{r})=[\nabla \otimes \nabla-\nabla \times(\nabla \times \widetilde{\mathbf{I}})] \cdot \widetilde{\mathbf{H}}(\mathbf{r}) \cdot[\overleftarrow{\nabla} \otimes \overleftarrow{\nabla}-(\widetilde{\mathbf{I}} \times \overleftarrow{\nabla}) \times \overleftarrow{\nabla}],
$$

which expanded leads to the required decomposition as

$$
\begin{aligned}
\widetilde{\mathbf{F}}(\mathbf{r})=[\nabla \otimes \nabla] \cdot \widetilde{\mathbf{H}}(\mathbf{r}) \cdot[\overleftarrow{\nabla} \otimes \overleftarrow{\nabla}]-[\nabla \otimes \nabla] \cdot[\widetilde{\mathbf{H}}(\mathbf{r}) \times \overleftarrow{\nabla}] \times \overleftarrow{\nabla} \\
-\nabla \times[\nabla \times \widetilde{\mathbf{H}}(\mathbf{r})] \cdot[\overleftarrow{\nabla} \otimes \overleftarrow{\nabla}]+\nabla \times[\nabla \times \widetilde{\mathbf{H}}(\mathbf{r}) \times \overleftarrow{\nabla}] \times \overleftarrow{\nabla} .
\end{aligned}
$$

This means that a dyadic function can be expressed in terms of a scalar potential $\psi$, two vector potentials $\mathbf{g}_{1}, \mathbf{g}_{2}$, and a dyadic potential $\widetilde{\mathbf{G}}$ in the form

$$
\widetilde{\mathbf{F}}(\mathbf{r})=\nabla \otimes \nabla \psi(\mathbf{r})+\nabla \otimes\left[\mathbf{g}_{1}(\mathbf{r}) \times \overleftarrow{\nabla}\right]+\left[\nabla \times \mathbf{g}_{2}(\mathbf{r})\right] \otimes \overleftarrow{\nabla}+\nabla \times \widetilde{\mathbf{G}}(\mathbf{r}) \times \overleftarrow{\nabla}
$$

The four potential functions $\psi, \mathbf{g}_{1}, \mathbf{g}_{2}$, and $\widetilde{\mathbf{G}}$ can be expressed in terms of the dyadic field $\widetilde{\mathbf{H}}$ as

$$
\begin{aligned}
\psi(\mathbf{r}) & =\nabla \cdot \widetilde{\mathbf{H}}(\mathbf{r}) \cdot \overleftarrow{\nabla} \\
\mathbf{g}_{1}(\mathbf{r}) & =-\nabla \cdot \widetilde{\mathbf{H}}(\mathbf{r}) \times \overleftarrow{\nabla} \\
\mathbf{g}_{2}(\mathbf{r}) & =-\nabla \times \widetilde{\mathbf{H}}(\mathbf{r}) \cdot \overleftarrow{\nabla} \\
\widetilde{\mathbf{G}}(\mathbf{r}) & =\nabla \times \widetilde{\mathbf{H}}(\mathbf{r}) \times \overleftarrow{\nabla}
\end{aligned}
$$


However, without any further restrictions, the potential functions are not unique. For example, we can add to $\psi$ any expression of the form $\mathbf{c r}$, where $\mathbf{c}$ is an arbitrary constant, and (31) would still be valid. The representation can be made unique by imposing the following conditions for the two vector potentials:

$$
\nabla \cdot \mathbf{g}_{1}(\mathbf{r})=\nabla \cdot \mathbf{g}_{2}(\mathbf{r})=0
$$

which leaves us two independent scalar functions for each of $\mathbf{g}_{1}$ and $\mathbf{g}_{2}$. The dyadic potential $\widetilde{\mathbf{G}}$ satisfies the two vector conditions

$$
\nabla \cdot \widetilde{\mathbf{G}}(\mathbf{r})=\widetilde{\mathbf{G}}(\mathbf{r}) \cdot \overleftarrow{\nabla}=\mathbf{0}
$$

Writing $\widetilde{\mathbf{G}}$ in terms of its symmetric and antisymmetric parts as $[8,13]$

$$
\widetilde{\mathbf{G}}(\mathbf{r})=\widetilde{\mathbf{G}}_{s}(\mathbf{r})+\mathbf{g}(\mathbf{r}) \times \widetilde{\mathbf{I}},
$$

where $\mathbf{g}$ is another vector potential, (38) is split into two parts as

$$
\begin{gathered}
\nabla \cdot \widetilde{\mathbf{G}}_{s}(\mathbf{r})=\mathbf{0}, \\
\nabla \cdot(\mathbf{g}(\mathbf{r}) \times \widetilde{\mathbf{I}})=\nabla \times \mathbf{g}(\mathbf{r})=\mathbf{0} .
\end{gathered}
$$

Because $\widetilde{\mathbf{G}}_{s}$ has originally six scalar potentials, (40) reduces them by three. The condition (41) leaves only one free scalar function for the vector potential $\mathbf{g}$. Thus, instead of $1+3+3+9=16$ potentials in the decomposition (32) we are left with only $1+2+2+3+1=$ 9 independent scalar potentials which, again, coincides with the dimension of the dyadic field.

Let us briefly consider the decomposition for two special dyadic functions. Splitting the dyadic equation (27) into its symmetric and antisymmetric part (by taking the sum and the difference of (27) and its transpose), we see that for a symmetric dyadic field $\widetilde{\mathbf{F}}_{s}$ there corresponds a symmetric dyadic field $\widetilde{\mathbf{H}}_{s}$, and for an antisymmetric dyadic $\widetilde{\mathbf{F}}_{a}$ an antisymmetric dyadic $\widetilde{\mathbf{H}}_{a}$ can be assigned. Both dyadics $\widetilde{\mathbf{H}}_{s}$ and $\widetilde{\mathbf{H}}_{a}$ are solutions of Eq. (27).

The decomposition for $\widetilde{\mathbf{F}}_{s}$ is of the form (32) and the potential $\widetilde{\mathbf{G}}$ is a symmetric dyadic, as is seen from (36). In this case, we arrive at

$$
\mathbf{g}_{1}(\mathbf{r})=-\left[\nabla \cdot \widetilde{\mathbf{H}}_{s}(\mathbf{r})\right] \times \overleftarrow{\nabla}=\nabla \times\left[\nabla \cdot \widetilde{\mathbf{H}}_{s}(\mathbf{r})\right]=\nabla \times\left[\widetilde{\mathbf{H}}_{s}(\mathbf{r}) \cdot \overleftarrow{\nabla}\right]=-\mathbf{g}_{2}(\mathbf{r}),
$$

whence the two vector potentials are simply related. From the restrictions (37) we now see that only two independent potentials define the vector potential $\mathbf{g}_{1}=-\mathbf{g}_{2}$. Because the two restrictions in (38) are now the same, there are only $6-3=3$ independent potentials for the symmetric dyadic $\widetilde{\mathbf{G}}$ and, thus, in total, the number of independent potentials is equal to $1+2+3=6$, the dimension of the symmetric dyadic $\widetilde{\mathbf{F}}_{s}$.

Because any antisymmetric dyadic $\widetilde{\mathbf{A}}$ can be expressed in terms of a vector in the form $\widetilde{\mathbf{A}}=\mathbf{a} \times \widetilde{\mathbf{I}}[8,13]$, we can write the antisymmetric dyadic field as $\widetilde{\mathbf{F}}_{a}=\mathbf{f} \times \widetilde{\mathbf{I}}$ and the corresponding antisymmetric dyadic solution of (27) as $\widetilde{\mathbf{H}}_{a}=\mathbf{h} \times \widetilde{\mathbf{I}}$, where $\mathbf{f}, \mathbf{h}$ are 
vector fields. From (33)-(36) we now have (for rules of dyadic operations see [13])

$$
\begin{gathered}
\psi(\mathbf{r})=\nabla \cdot[\mathbf{h}(\mathbf{r}) \times \widetilde{\mathbf{I}}] \cdot \overleftarrow{\nabla}=-\nabla \cdot \nabla \times \mathbf{h}(\mathbf{r})=0, \\
\mathbf{g}_{1}(\mathbf{r})=-\nabla \cdot[\mathbf{h}(\mathbf{r}) \times \widetilde{\mathbf{I}}] \times \overleftarrow{\nabla}=-[\nabla \times \mathbf{h}(\mathbf{r})] \times \overleftarrow{\nabla}=\nabla \times \nabla \times \mathbf{h}(\mathbf{r}), \\
\mathbf{g}_{2}(\mathbf{r})=-\nabla \times[\mathbf{h}(\mathbf{r}) \times \widetilde{\mathbf{I}}] \cdot \overleftarrow{\nabla}=-\nabla \times[\mathbf{h}(\mathbf{r}) \times \overleftarrow{\nabla}]=\nabla \times \nabla \times \mathbf{h}(\mathbf{r})=\mathbf{g}_{1}(\mathbf{r}), \\
\widetilde{\mathbf{G}}(\mathbf{r})=\nabla \times[\mathbf{h}(\mathbf{r}) \times \widetilde{\mathbf{I}}] \times \overleftarrow{\nabla}=-[\nabla \cdot \mathbf{h}(\mathbf{r})] \widetilde{\mathbf{I}} \times \overleftarrow{\nabla}=-\nabla[\nabla \cdot \mathbf{h}(\mathbf{r})] \times \widetilde{\mathbf{I}}
\end{gathered}
$$

Thus, (32) can be written in the form

$$
\begin{aligned}
\widetilde{\mathbf{F}}_{a}(\mathbf{r})= & \mathbf{f}(\mathbf{r}) \times \widetilde{\mathbf{I}} \\
= & \nabla \otimes\left[-\nabla^{2} \mathbf{h}(\mathbf{r}) \times \overleftarrow{\nabla}\right]+\left[-\nabla^{2} \nabla \times \mathbf{h}(\mathbf{r})\right] \otimes \overleftarrow{\nabla} \\
& +\nabla \times[-\nabla(\nabla \cdot \mathbf{h}(\mathbf{r})) \times \widetilde{\mathbf{I}}] \times \overleftarrow{\nabla} \\
= & \nabla\left[\nabla^{2} \nabla \cdot \mathbf{h}(\mathbf{r})\right] \times \widetilde{\mathbf{I}}-\nabla \times\left[\nabla^{2} \nabla \times \mathbf{h}(\mathbf{r})\right] \times \widetilde{\mathbf{I}} .
\end{aligned}
$$

Identifying the vectors of the antisymmetric dyadics we obtain the decomposition

$$
\mathbf{f}(\mathbf{r})=\nabla \phi(\mathbf{r})+\nabla \times \mathbf{A}(\mathbf{r})
$$

where the scalar and vector potentials are defined as

$$
\begin{gathered}
\phi(\mathbf{r})=\nabla^{2} \nabla \cdot \mathbf{h}(\mathbf{r}), \\
\mathbf{A}(\mathbf{r})=-\nabla^{2} \nabla \times \mathbf{h}(\mathbf{r}) .
\end{gathered}
$$

The decomposition (48) recovers the original Helmholtz decomposition (6) for vector fields.

3. Decomposition of polyadics. The representations (18) and (20) can easily be generalized to polyadic fields of any rank. Indeed, linearity and straightforward induction imply that the $n$th rank polyadic field

$$
\widetilde{\mathbf{F}}^{(n)}(\mathbf{r})=\mathbf{f}_{1}(\mathbf{r}) \otimes \mathbf{f}_{2}(\mathbf{r}) \otimes \cdots \otimes \mathbf{f}_{n}(\mathbf{r})=\bigotimes_{k=1}^{n} \mathbf{f}_{k}(\mathbf{r})
$$

can be decomposed as

$$
\widetilde{\mathbf{F}}^{(n)}(\mathbf{r})=\nabla \otimes \widetilde{\mathbf{P}}_{n-1}^{(n-1)}(\mathbf{r})+\nabla \times \widetilde{\mathbf{P}}_{n}^{(n)}(\mathbf{r})
$$

where $\widetilde{\mathbf{P}}_{n-1}^{(n-1)}$ is a polyadic potential of rank $(n-1)$ and $\widetilde{\mathbf{P}}_{n}^{(n)}$ is a polyadic of rank $n$. The field $\widetilde{\mathbf{F}}^{(n)}$ has $3^{n}$ components and the condition

$$
\nabla \cdot \widetilde{\mathbf{P}}_{n}^{(n)}(\mathbf{r})=\widetilde{\mathbf{0}}^{(n-1)}
$$

makes the independent components of $\widetilde{\mathbf{P}}_{n}^{(n)}$ to $3^{n}-3^{n-1}=2 \cdot 3^{n-1}$. Hence, the number of independent scalar potentials in the decomposition (51) is again $3^{n-1}+2 \cdot 3^{n-1}=3^{n}$. 
Repeating the corresponding argument of the previous section we arrive at the complete decomposition

$$
\begin{aligned}
\widetilde{\mathbf{F}}^{(n)}(\mathbf{r})= & \nabla \otimes \nabla \otimes \cdots \otimes \nabla P_{0}(\mathbf{r}) \\
& +\nabla \otimes \nabla \otimes \cdots \otimes \nabla \times \mathbf{P}_{1}(\mathbf{r}) \\
& +\nabla \otimes \nabla \otimes \cdots \otimes \nabla \times \nabla \times \widetilde{\mathbf{P}}_{2}^{(2)}(\mathbf{r}) \\
& +\cdots \\
& +\nabla \otimes \nabla \times \cdots \times \nabla \times \widetilde{\mathbf{P}}_{n-1}^{(n-1)}(\mathbf{r}) \\
& +\nabla \times \nabla \times \cdots \times \nabla \times \widetilde{\mathbf{P}}_{n}^{(n)}(\mathbf{r}) .
\end{aligned}
$$

In the representation (54) the first term involves $n$ successive applications of the gradient operator on a scalar potential, the second term involves $(n-1)$ successive applications of the gradient to the rotation of a vector potential, and so on. As we move from term to term, we replace the action of a gradient by the rotation of a potential of one rank higher. The last term involves $n$ successive rotations upon a polyadic potential of rank $n$. Hence, all combinations of gradients and rotations are taken.

The generalized Helmholtz representation (54) involves $(n+1)$ terms, where $n$ stands for the rank of the polyadic that is decomposed. For $n=1$ we are dealing with vector fields and the representation involves two terms, i.e., the Helmholtz decomposition formula (6).

If we decompose the potential $\widetilde{\mathbf{P}}_{n}^{(n)}$ by means of (54), we see that all but the last term of the decomposition (the one involving only curl operators) can be taken as zero, because the rotation of any polyadic that starts with a gradient vanishes. This remark keeps the number of independent scalar potentials equal to $3^{n}$ and can be imposed as

$$
\begin{gathered}
\nabla \cdot \widetilde{\mathbf{P}}_{n}^{(n)}(\mathbf{r})=\widetilde{\mathbf{0}}^{(n-1)}, \\
\nabla \otimes \nabla: \widetilde{\mathbf{P}}_{n}^{(n)}(\mathbf{r})=\widetilde{\mathbf{0}}^{(n-2)}, \\
\cdots \\
\nabla \otimes \nabla \otimes \cdots \otimes \nabla \cap \widetilde{\mathbf{P}}_{n}^{(n)}(\mathbf{r})=0,
\end{gathered}
$$

where the symbol (n) (" $n$-dot product") indicates $n$ successive contractions in the sense

$$
\left(\bigotimes_{i=1}^{n} \mathbf{a}_{i}\right) \cap\left(\bigotimes_{i=1}^{n} \mathbf{b}_{i}\right)=\prod_{i=1}^{n}\left(\mathbf{a}_{i} \cdot \mathbf{b}_{i}\right) \text {. }
$$

\section{REFERENCES}

[1] L. Brand, Vector and Tensor Analysis, John Wiley and Sons, New York, London, 1947

[2] P. Chadwick and E. A. Trowbridge, Elastic wave fields generated by scalar wave functions, Proc. Cambridge Philos. Soc. 63, 1177-1187 (1967)

[3] P. Ciarlet, A decomposition of $L^{2}(\Omega)^{3}$ and an application to magnetostatic equations, Math. Mod. Meth. Appl. Sci. 3, 289-301 (1993)

[4] G. Dassios and R. Kleinman, Low Frequency Scattering, Oxford University Press, 2000

[5] G. Dassios, K. Kiriaki, and D. Polyzos, Scattering theorems for complete dyadic fields, Internat. J. Engrg. Sci. 33, 269--277 (1995)

[6] R. A. Eubanks and E. Sternberg, On the completeness of the Boussinesq-Papkovich stress functions, J. Rat. Mech. Anal. 5, 735-746 (1956) 
[7] B. Galerkin, Contribution à la solution générale du problème de la théorie de l'élasticité dans la cas de trois dimensions, Compt. Rend. 190, 1047-1048 (1930)

[8] J. W. Gibbs, Vector Analysis, Yale University Press, New Haven, 1901

[9] M. E. Gurtin, The linear theory of elasticity, in Encyclopaedia of Physics (C. Truesdell, editor), Vol. VIa/2, Springer-Verlag, Berlin, 1972

[10] R. P. Kanwal, The existence and completeness of various potentials for the equations of Stokes flow, Internat. J. Engrg. Sci. 9, 375-386 (1971)

[11] J. B. Keller, Simple proofs of the theorems of J. S. Lomont and H. E. Moses on the decomposition and representation of vector fields, Comm. Pure Appl. Math. 14, 77-80 (1960)

[12] W. Kratz, On the representation of Stokes flows, SIAM J. Math. Anal. 22, 414-423 (1991)

[13] I. V. Lindell, Methods for Electromagnetic Field Analysis, 2nd ed., Oxford University Press, 1995

[14] J. S. Lomont and H. E. Moses, An angular momentum Helmholtz theorem, Comm. Pure Appl. Math. 14, 69-76 (1960)

[15] J. Mathews, Gravitational multipole radiation, J. Soc. Indust. Appl. Math. 10, 768-780 (1962)

[16] H. Neuber, Ein neuer Ansatz zur Lösung räumlicher Probleme der Elastizitätstheorie, Z. Angew. Math. Mech. 14, 203-212 (1934)

[17] D. Palaniappan, S. D. Nigam, T. Amaranath, and R. Usha, Lamb's solution of Stokes's equations: A sphere theorem, Quart. J. Mech. Appl. Math. 45, 47-56 (1992)

[18] P. F. Papkovich, The representation of the general integral of the fundamental equations of elasticity theory in terms of harmonic functions, Izv. Akad. Nauk SSSR, Ser. Mat. 10, 1425-1435 (1932) (in Russian)

[19] P. F. Papkovich, Solution générale des équations différentielles fondamentales d'élasticité exprimée par trois fonctions harmoniques, Compt. Rend. 195, 513-515 (1932)

[20] D. A. W. Pecknold, On the role of the Stokes-Helmholtz decomposition in the derivation of displacement potentials in classical elasticity, J. Elast. 1, 171-174 (1971)

[21] E. Sternberg, On the integration of the equations of motion in the classical theory of elasticity, Arch. Rat. Mech. Anal. 6, 34-50 (1960)

[22] T. Tran-Cong and J. R. Blake, General solutions of the Stokes' flow equations, J. Mat. Anal. Appl. 90, 72-84 (1982)

[23] X. Xinsheng and W. Minzhong, General complete solutions of the equations of spatial and axisymmetric Stokes flow, Quart. J. Mech. Appl. Math. 44, 537-548 (1991)

[24] D. A. Woodside, Uniqueness theorems for classical four-vector fields in Euclidean and Minkowski spaces, J. Math. Phys. 40, 4911-4943 (1999) 